Clio. Femmes, Genre, Histoire

1 | 1995

Résistances et Libérations France 1940-1945

\title{
Fantasmes du temps de la Libération
}

\section{Geneviève SELLIER et Noël BURCH}

\section{(2) OpenEdition}

Journals

Édition électronique

URL : https://journals.openedition.org/clio/522

DOI : $10.4000 /$ clio.522

ISSN : 1777-5299

Éditeur

Belin

Édition imprimée

Date de publication : 1 avril 1995

ISSN : 1252-7017

Référence électronique

Geneviève SELLIER et Noël BURCH, «Fantasmes du temps de la Libération », Clio. Femmes, Genre, Histoire [En ligne], 1 | 1995, mis en ligne le 01 janvier 2005, consulté le 24 avril 2022. URL : http:// journals.openedition.org/clio/522 ; DOI : https://doi.org/10.4000/clio.522

Ce document a été généré automatiquement le 24 avril 2022

Tous droits réservés 


\title{
Fantasmes du temps de la Libération
}

\author{
Geneviève SELLIER et Noël BURCH
}

1 L'approche historique des films souffre en France d'une double myopie : soit on sauve selon des critères cinéphiliques hérités de la "politique des auteurs » quelques chefsd'œuvre qui transcendent leur époque; soit on évalue les films selon des critères politiques ou idéologiques exogènes, comme «reflets» des débats de l'heure. Nous avons tenté d'élaborer des hypothèses à partir de ce dont parlent la plupart des films de fiction en France : les relations entre les hommes et les femmes.

2 Or l'analyse des représentations des rapports hommes / femmes dans le cinéma de fiction de l'après-guerre ${ }^{1}$ fait apparaitre l'épisode des tondues et de la recrudescence des divorces à la Libération comme une partie (à peine) visible d'un iceberg: celui d'une crise profonde des rapports sociaux de sexe dont l'équilibre traditionnel a été ébranlé par la défaite militaire et les nouvelles responsabilités prises par les femmes sous l'Occupation. Malgré le droit de vote (enfin) accordé aux femmes en 1945, le discours politique de l'après-guerre, de droite comme de gauche, va se contenter de remettre en place, à quelques nuances près, la traditionnelle division sexuelle des rôles sociaux, mais les productions culturelles et en particulier cinématographiques témoignent abondamment de la crise que tente de recouvrir ce consensus.

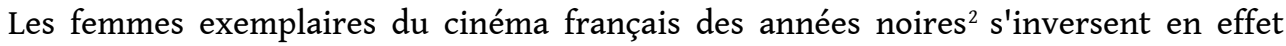
brutalement dans un déferlement d'images féminines diaboliques, dont on peut pointer le modèle dans Manèges d'Yves Allégret (1949), et trouver encore, en 1956 dans Voici le temps des assassins de Duvivier, une paroxystique illustration, pendant qu'émergent des représentations, minoritaires certes, mais d'une vigueur sans précédent, de femmes " en émancipation ", comme dans Antoine et Antoinette de Becker (1946), Le Carrosse d'or de Renoir (1952) ou l'Amour d'une femme de Grémillon (1954).

4 Les films sur la Résistance se trouvent massivement du côté de la misogynie, en en donnant une image exclusivement masculine, comme dans La Bataille du rail (René Clément, 1945), Un ami viendra ce soir (Raymond Bernard, 1945), Les Démons de l'aube (Yves Allégret, 1945), Patrie (Daquin, 1945), ou Jéricho (H. Calef, 1945).

5 Le grand succès commercial de l'immédiat après-guerre, c'est Le Père tranquille (1946) de Noël-Noël (réalisation technique de René Clément). Monsieur Martin (Noël-Noël), 
quintessence du Français moyen, ancien combattant sept fois décoré de la guerre précédente, passe auprès de sa famille pour un homme gentil mais un peu lâche, uniquement préoccupé de ses orchidées alors que les Allemands occupent la France. Mais bientôt le spectateur, puis les enfants de cet «anti-héros » s'apercevront de leur erreur, découvrant derrière ce vieil homme frileux le chef de la Résistance de toute la région.

6 La réalisation assez terne est au service exclusif de la reconstruction de l'image du père, comme s'il fallait persuader les jeunes et les femmes - les autres personnages masculins, eux, ne sont pas dupes, puisqu'ils participent à la Résistance sous les ordres de Monsieur Martin - que tous les anciens combattants ne sont pas à l'image du Maréchal Pétain... Le film tente de nous faire croire que l'attentisme, dont Pierre Laborie a montré qu'il fut l'attitude majoritaire des Français, était le meilleur camouflage de l'esprit résistant. Cette nouvelle version de «l'habit ne fait pas le moine » vient opportunément dédouaner la «majorité silencieuse » de sa passivité pendant les années noires... Mais les profits et pertes de cette opération ne sont pas uniformément partagées: la cible privilégiée du Père tranquille est la femme de ce Français moyen, mère de famille engluée dans le quotidien, incapable de voir plus loin que le repas du soir et la potion contre la toux. Quant à la fille (Nadine Alari), elle passe d'une tendresse indulgente à l'égard d'un père qu'elle croit faible à une admiration sans borne pour celui qu'elle découvre comme le grand chef de la Résistance. Elle se mariera avec le lieutenant de ce père adoré qui lui a fait l'immense faveur de la mettre dans la confidence de ses activités clandestines. Le couple père / fille, mis à mal pendant l'Occupation, est ainsi reconstitué comme si de rien n'était !

7 Enfin dernier enjeu du film, les relations père-fils : ce jeune adolescent irresponsable qui prend son père pour un lâche, part au maquis "pour qu'il y ait au moins une personne de la famille qui fasse de la Résistance »; on le retrouve enfermé par les maquisards dans la cabane à cochon (!) pour l'empêcher de faire des bêtises ; il en sort pour retrouver son père blessé après son arrestation par la Gestapo et sa délivrance par les hommes du maquis. Les larmes du fils témoignent de son repentir. On peut lire en creux, dans ce fantasme d'un fils révolté qui fait amende honorable, la réalité de la crise de l'autorité paternelle qui éclate à la Libération, quand toute une génération de pères prétend reprendre en main des fils laissés à eux-mêmes pendant cinq ans.

8 L'intérêt principal de ces œuvres de circonstance est de proposer (d'imposer) une vérité officielle de la période précédente $^{3}$ où le triomphe de la Résistance, exclusivement incarnée par des hommes, est inséparable du retour des femmes à « leur » place de dominées.

9 Les espoirs de révolution sociale issus de la Résistance et de la Libération s'essouflèrent rapidement face aux réalités politiques et économiques, nationales et internationales de l'après-guerre; le courant du "réalisme noir» au cinéma peut être interprété comme la «traduction» de ces désillusions idéologiques; mais une telle analyse ne rend pas compte de la problématique dominante de ces films, qui les différencie de la noirceur du « réalisme poétique » d'avant-guerre : l'élément nouveau est l'articulation entre le caractère (objectivement ou subjectivement) maléfique des femmes et la victimisation des hommes. La "fatalité » s'incarne maintenant, non plus sous les traits repérables d'un bourgeois vicieux (cf. Jules Berry dans Le Crime de Monsieur Lange ou Le Jour se lève), mais sous l'apparence trompeuse d'une belle jeune femme: la problématique sociale d'avant-guerre a fait place à une thématique de guerre des sexes, 
où les femmes ont une emprise destructrice sur les hommes. On trouve ce schéma dans Une si jolie petite place et Manèges (Yves Allégret, 1948 et 1949), Quai des Orfêvres et Manon (Clouzot, 1947 et 1948), Panique et Voici le temps des assassins (Duvivier, 1946 et 1956), Un Revenant (Christian-Jaque, 1946)...

10 L'insistance mise sur la faiblesse masculine, face à des personnages féminins d'une force souvent ravageuse, peut se lire comme l'interprétation paranoïde que font les hommes de leur situation à la Libération, dans l'angoisse de ne pas retrouver leur domination d'avant-guerre, et vivant l'émancipation des femmes comme une tentative de destruction de l'identité masculine.

11 Quai des Orfêvres (1947) permet à Clouzot de refaire brillamment surface après avoir purgé deux ans d'interdiction professionnelle. La malfaisance des femmes, voilà un thème éminemment consensuel qui permet d'oublier les divergences idéologiques et les comportements douteux... Suzy Delair trouve sans doute le meilleur rôle de sa carrière dans le personnage de Jenny Lamour, chanteuse de music-hall dont l'arrivisme sans frein amène son mari à commettre un meurtre (ou presque). Bernard Blier incarne «naturellement» ce mari amoureux et dupe, gentil et faible, petit-bourgeois déclassé par amour, pianiste dans le music hall où se produit sa femme, réduit le plus souvent à assister impuissant à ses entreprises de séduction. Incarnation d'une autorité patriarcale lucide mais démonétisée, le commissaire Jouvet fait son enquête, pour découvrir, la mort dans l'âme, que le pauvre mari est sans doute le coupable, alors que sa "garce » de femme est la seule responsable! Mais pour qu'il ne soit pas dit que toutes les femmes sont des "garces", Simone Renant incarne une photographe lesbienne, secrètement amoureuse elle aussi de la belle Jenny, et prête à se compromettre pour réparer ses imprudences (ou son crime), et que Jouvet qualifie avec sympathie de "type dans (son) genre». Ainsi les hommes et les lesbiennes sont présentés comme les victimes de la séduction féminine et supportent courageusement leur condition malheureuse.

12 Par ailleurs, Bernard Blier et Louis Jouvet donnent chacun à leur façon une image fragile et sympathique de la masculinité : le jeune mari, sans défense face à l'arrivisme de sa femme, et le vieil inspecteur revenu des colonies avec le paludisme et un enfant métis qu'il couve comme une mère. Deux incarnations de la Loi patriarcale très efficacement camouflée que le cinéma de l'après-guerre s'attache sans relâche à réhabiliter : si Jenny Lamour avait obéi à son mari, ce cauchemar n'aurait pas eu lieu ; et seule la persévérance tatillonne de l'inspecteur finit par innocenter le mari, après avoir complètement détruit ses alibis. Charles Dullin, l'horrible vieillard libidineux, concession à l'idée d'un patriarcat abusif, est en fait très dérisoire, se contentant de faire photographier ses conquêtes dans des poses lascives, parce qu'il n'a plus la force de les " posséder ».

13 En épargnant in extremis celle qui reste moralement la grande coupable, le film se paye le luxe de paraître magnanime... Jouvet, porte-parole des auteurs, vient donner son absolution au jeune couple, mais il est clair pour le spectateur que c'est la générosité du mari qui mérite d'être récompensée. Le choix de Bernard Blier, avec sa laideur banale de "Français moyen", renforce l'identification du spectateur, mais aussi de la spectatrice qui a bien du mal en revanche à se trouver des points communs avec la chanteuse de cabaret provocante et irresponsable, ou avec la lesbienne solitaire.

14 L'efficacité de Quai des orfêvres, couronné au Festival de Venise de 1947, tient au caractère extrêmement brillant de la mise en scène, depuis la construction du récit 
jusqu'à la direction d'acteurs, dans un contraste fascinant avec la médiocrité des personnages et le caractère sordide du fait divers, contraste qui deviendra la « signature » de Clouzot.

Mais la déferlante misogyne de l'immédiat après-guerre n'est pas l'expression unique, bien que largement majoritaire, des bouleversements provoqués par la défaite et l'Occupation dans les rapports entre les sexes. Comme un contrepoint obstiné, et souvent remarquable, émerge ce qu'on pourrait appeler un véritable courant féministe dans le cinéma français, dont la nouveauté par rapport à la période précédente apparaît nettement: on passe de figures féminines positives, mais souvent idéalisée, à des représentations qui se confrontent audacieusement aux contradictions mises à jour par la Libération dans les relations entre hommes et femmes; les plus novateurs de ces films abordent à la fois la question du désir - libérateur ou aliénant -, les rapports de couple, l'articulation entre sphère professionnelle et sphère " privée " pour chacun des deux sexes, le poids de l'éducation dans le comportement des uns et des autres, enfin tout ce qui fait que les rapports entre les sexes s'inscrivent dans une histoire et dans une société données. C'est sans doute cette tentative - plus ou moins aboutie selon les films - de questionner des rapports si longtemps donnés comme « naturels », qui fait la nouveauté et la modernité de ce courant, à mettre en perspective avec Le Deuxième Sexe de Simone de Beauvoir publié en 1949.

Ce courant, bien que minoritaire, va s'exprimer chez les cinéastes les plus divers, artistiquement et idéologiquement parlant, ce qui confirme la force qu'a pris cette question dans la société française de l'après-guerre. Certains de ces films émanent des milieux résistants ou progressistes - comme Antoine et Antoinette de Jacques Becker (1946), Les Frères Bouquinquant (1947) et Le Point du Jour (1948) de Louis Daquin, Pattes Blanches de Jean Grémillon (1948), Le Silence de la mer du jeune Jean-Pierre Melville (1947) et enfin Les Malheurs de Sophie (1946), Gigi (1948) et Minne, l'ingénue libertine (1950) de Jacqueline Audry qui a fait ses classes de cinéaste dans le maquis. Mais ce courant féministe atteint aussi des cinéastes restés à l'écart de la lutte comme Jean Cocteau ( $L a$ Belle et la Bête, 1945), Christian-Jaque (Boule de suif, 1945), Henri Decoin (Les Amants du Pont Saint-Jean, 1947).

Cette veine va s'amenuiser au cours des années cinquante, mais continuera de produire des films intéressants ou remarquables (Casque d'or, Le Carrosse d'or, La Vérité sur Bébé Donge, L'Amour d'une femme, Madame de..., Lola Montès).

Casque d'or (1952), scénario qui date d'avant-guerre et sur lequel Becker commence à travailler dès 1946, raconte, sur fond de Belle époque, l'histoire d'amour entre Manda le menuisier (Serge Reggiani) et Marie la grisette (Simone Signoret) : c'est elle qui le séduit dans une guinguette, le relance dans sa boutique, envoie balader son souteneur, et finit par défier même le chef de la bande (Claude Dauphin), pour faire respecter sa liberté. L'homme qu'elle choisit d'aimer est "l'antimacho" par excellence; le travail artisanal, tel que Manda le pratique avec une modeste efficacité, est l'exacte antithèse de l'oisiveté agressive des petits malfrats qui accompagnent Marie et ses amies. Petit et silencieux, le menuisier accueille avec une tranquillité souriante toutes les initiatives de Marie et ne fait usage de sa force qu'en cas de légitime défense. En face de cet « homme nouveau » qui n'a pas besoin de dominer (en particulier les femmes) pour se sentir exister, "Casque d'or ", avec son allure à la fois familière et conquérante, est le symbole éclatant des femmes qui décident de vivre debout, quoiqu'il en coûte. Mais ici le désir amoureux féminin déclenche la volonté d'émancipation, au lieu de la détourner 
comme dans la plupart des films des années cinquante. Le pouvoir patriarcal, superbement incarné dans sa violence perverse par le chef de bande Claude Dauphin, doit détruire à la fois l'homme doux et la femme rebelle, comme deux exemples subversifs d'une société non-sexiste et non-exploiteuse. La force du film est dans l'articulation des affrontements de classe et de sexe ${ }^{4}$.

Ainsi le cinéma témoigne, comme aucun autre moyen d'expression nous semble-t-il, de la déstabilisation des rapports entre les sexes dans la société française des années 1940, provoquée certes par les événements politiques et militaires, mais étudiée ici au travers de ses répercussions dans le champ de la vie privée, traditionnellement considérée comme relevant d'une autre temporalité («la longue durée» de l'histoire des mentalités). La sensibilité du cinéma aux bouleversements induits, dans le champ des rapports sociaux de sexe, par la guerre et l'Occupation prouvent à quel point la sphère privée est politique...

Par ailleurs, la mise au jour d'une strate de significations, visible seulement (à ce jour) dans ce champ partiel de la production culturelle qu'est le cinéma de fiction, pourrait aussi montrer l'intérêt d'aborder l'histoire du cinéma comme un champ autonome, qui peut nous apprendre beaucoup plus sur l'état de nos sociétés, dès lors qu'on ne considère plus les films comme un simple reflet, fût-il à contre-courant, d'idées élaborées ailleurs.

\section{NOTES}

1. N. Burch et G. Sellier, La Drôle de guerre des sexes du cinéma français (1930-1955), à paraître chez Nathan en 1995 ; « Guerre des sexes dans le cinéma français : les turbulences de l'après-guerre ", in Les Cahiers de l'IHTP, février 1995.

2. N. Burch et G. Sellier, « Rencontre avec des femmes remarquables : représentation des relations sociales de sexes dans le cinéma français sous l'Occupation, 1940-1944 ", in Projets féministes $\mathrm{n}^{\circ}$ 3, octobre 1994 ; et « Les pères châtrés du cinéma de l'Occupation ", in Génériques, n 1, hiver 1995.

3. Voir aussi Sylvie Lindeperg, Images de la seconde guerre mondiale dans le cinéma français (1944-1969), Thèse de doctorat, Institut d'études politiques, 1993.

4. G. Sellier, « L'après-guerre : contradictions d'un auteur », in La Pensée, $n^{\circ} 300$, oct.nov.-déc. 1994. 